\title{
MATHEMATICAL MODELS OF FILTRATION PROCESSES VIDEO IMAGES
}

\author{
E.B.Tashmanov ${ }^{1}$ \\ ${ }^{I}$ Tashkent University of Information Technologies, Uzbekistan
}

\begin{abstract}
Possibility of improving the images quality which contains interfering video objects by removing them with proposed algorithm based on Chebyshev polynomials is considered in the article. The algorithm work and the accuracy results of pixels values recovery on the basis of experimental software is given in the article.
\end{abstract}

Keywords: Image, Chebyshev Polynomials, Disturbing Video Objects, Image Reconstruction, The Accuracy of Recovery Pixels.

\section{INTRODUCTION}

Digital images are exposed to different types of noise that can occur in the sensor imaging, information transmission technology, and data digitization methods. The process of silencing in the images is called filtration.

While filtering the brightness parameter of each digital image, point is replaced with another brightness value that is recognized as lesser extent distorted disturbance.

In practice image processing by various filters is applied in order to correct small defects and noise (Wiener, the median filter, Sobel operator and etc.).

Image processing by such filters eliminates the effect of noise very well, improves image sharpness, but it cannot cope with large blotches. It is especially important in applied video information systems when it often causes a problem in removing disturbing video objects. Therefore, adaptive methods and algorithms are required to improve images quality with such structure in the preprocessing phase.

\section{DEVELOPED A MODEL OF FILTRATION}

\section{PROCESSES}

Differential operators of higher order, such as the Laplace operator can be used to eliminate defects or disturbing video objects in the images: [1]

$$
\frac{\partial^{2} z}{\partial x^{2}}+\frac{\partial^{2} z}{\partial y^{2}}=f(x, y)
$$

where, $z=z(\mathrm{x}, y)$-brightness function, $z_{i, j}-$ the brightness at the point $\left(\mathrm{x}_{i}, \mathrm{y}_{j}\right)$.

In order to apply this equation (1) in the digital image processing, it is necessary to express it in a discrete form.

$$
-4 z_{i, j}+z_{i-1, j}+z_{i+1, j}+z_{i, j-1}+z_{i, j+1}=f\left(x_{i}, y_{j}\right)
$$

Equation (2) can be used to improve image quality, but it cannot cope with large blotches, as a small dressing filters are used to reduce the distortions of video objects.

In order to correct the large blotches in the image, this area can be selected and brightness (tone) of each original image point of distorted disturbance can be changed. As a result of the correction, replacement of pixel values is made by other brightness value, which is recognized as the least distorted disturbance, i.e. brightness control of image pixels is made. In terms of above mentioned the control function should be added to the second equation instead of a fixed function. Thus, the mathematical model of above mentioned problem is as follows:

$$
-4 z_{i, j}+z_{i-1, j}+z_{i+1, j}+z_{i, j-1}+z_{i, j+1}=u\left(x_{i}, y_{j}\right)
$$

From the mathematical point of view the task was correctly, the area in which this task is considered should be defined, which is denoted by $\Omega$, and boundary conditions is also added, such as:

$$
\left.z\right|_{\partial \Omega}=\varphi
$$

Thus, the image border can also be controlled. However, control capability cannot be unlimited, so terminate it as the following form $|u| \leq \rho$.In this example, eight bit image has at maximum brightness value $\rho=256$ and ten bit image has $\rho=1024$. (3) and (4) are two-parameter discrete problem of control. In mathematics such control problems were less studied, and from the technological point of view as the application for image processing is not studied at all.

When transmitting television pictures brightness value at the same point of one frame does not usually coincide with the 
other frames. Noticed brightness variations must be taken into account at every point in solving these problems. This shows that the process involves party with opposite interest i.e., it prevents controlling the image brightness value.

The mathematical model of this problem is presented as follows: [2]

$$
\begin{gathered}
-4 z_{i, j}+z_{i-1, j}+z_{i+1, j}+z_{i, j-1}+z_{i, j+1}=u\left(x_{i}, y_{j}\right)-v\left(x_{i}, y_{j}\right) \\
z_{\partial \Omega}=\varphi
\end{gathered}
$$

(5), (6) describe a two-parameter discrete games of chase.

The chase is considered as complete if $z_{i j}$ satisfies the condition: $\quad \delta \leq z_{i, j} \leq \delta+\varepsilon, i_{0} \leq i \leq i_{1}, j_{0} \leq j \leq j_{1} \quad$ where $1 \leq i_{0}, i_{1} \leq m, 1 \leq \mathrm{j}_{0}, j_{1} \leq \theta-1$ for some predetermined $\delta>0, \varepsilon>0$.

It should be noted that the game refers to the process, which involves two or more parties, fighting for the realization of their interests. Each party has its own purpose and they use some strategy that can lead to the loss or gain which depends on the behavior of other players. Game theory helps to choose the best strategy taking into account the representations of the other participants, their resources and their possible actions. If the game is described by differential equations, it is called a differential game. As it's already stated, the problem (5), (6) describes a discrete game.

As a new discrete game the problem (5), (6), can be solved by using the Chebyshev polynomial of the second order. In this case the results are used as a mathematical tool for image processing and their mathematical model is represented as follows: [3-5]

$U_{n+2}(x)=2 x U_{n+1}(x)-U_{n}(x), n \geq 0$,

$U_{0}(x)=1, U_{1}(x)=2 x$

where: $U_{n+2}$ is the Chebyshev polynomial matrix, calculated at the next step $(n+2-$ the degree of the polynomial); $U_{n+1}$-Chebyshev polynomial matrix, which is calculated in the previous step $(n+2-$ the previous degree of the polynomial); $U_{n}$-Chebyshev polynomial matrix, which is calculated in two steps up to the current one; $x$-input parameter on the basis of it the polynomial is calculated.

$U_{-2}(X)=E, U_{-1}(X)=\overline{0}, U_{0}(X)=E, U_{1}(X)=2 X$, where $E$ is identity and $\overline{0}$ is zero matrix.

$$
\begin{aligned}
& z_{n}=U_{\theta-1}^{-1}\left(\frac{1}{2} C\right) U_{\theta-n-1}\left(\frac{1}{2} C\right)\left[z_{0}+\sum_{k=1}^{n-1} U_{k-1}\left(\frac{1}{2} C\right)\left(u_{k}-v_{k}\right)\right]+ \\
& +U_{\theta-1}^{-1}\left(\frac{1}{2} C\right) U_{n-1}\left(\frac{1}{2} C\right)\left[z_{\theta}+\sum_{k=n}^{\theta-1} U_{\theta-k-1}\left(\frac{1}{2} C\right)\left(u_{k}-v_{k}\right)\right] .
\end{aligned}
$$

where

$z_{0}$ and $z_{\theta}$ are the rows of source data for calculation correction factor and they are specified directly from an image (the first and last strings of subject area);

$U_{\theta-1}^{-1}$ - the inverse matrix of the Chebyshev polynomial matrix of $\theta-1$ degree; $U_{\theta-n-1}^{-1}-$ the Chebyshev polynomial matrix of $\theta-n-1$ degree; $C-$ Jacobian square matrix tridiagonal matrix, the argument for the calculation of Chebyshev polynomials $U_{n} ; U_{k-1}-$ the Chebyshev polynomial matrix of $k-1$ degree; $u_{k}$ and $v_{k}$ - control parameters in the form of vectors; $n$-row number in the correction factors matrix for the brightness matrix; $k-$ running index of the array sum; $\theta$-parameter that indicates the order of all matrix (brightness, correction) of command vectors.

This type of processing allows you to correct the pixels brightness in the image area by control.

Thus, by searching the control ratio (u, v), using the Chebyshev polynomials of the second kind (7) and (8) you can correct the brightness according to the specified range $(\beta, \beta+\varepsilon)$, where ( $\beta$ - the minimal brightness $\varepsilon$ - the difference between the maximum and minimum brightness, $z_{i j}$ - the brightness of the given point) within the working area. As a result, the measurement $\beta, \varepsilon$ is performed setting the brightness range $(\beta, \beta+\varepsilon)$, which should get all the points from the working area after correction.

Within the framework of determining the efficiency degree of image reconstruction algorithm several experiments have been carried out on two types - static and dynamic graphic information.

The scene "airship" presented in Figure 1 has been chosen in order to carry out a test. It contains four frames, the first is reference, and it represents a static image, and the next three ones are dynamic video scene. A certain amount of defects (5 to 7 ) were coated on every frame for simulating the real image damage. Root-mean-square deviation (RMSD) was used as a success measure of carried out restoration.

Five defects are identified in thisscene (Figure 1) and their coordinates of are given in Table 1. All defects have a size 
of 8 by 8 pixels and respectively the size of the describing area will be 10 by 10 pixels. Evaluation results of restoration accuracy in the background image on the basis of RMSD calculation are shown in Table 2, and in data bar in Figure 2.

Table -1: The coordinates of the upper left corner of the area with defects for the "Airship" plot

\begin{tabular}{|l|l|l|l|l|l|l|l|l|l|l|}
\hline & \multicolumn{3}{|l|}{ Province. 1 } & Pro. 2 & \multicolumn{2}{l|}{ Pro. 3 } & \multicolumn{2}{l|}{ Pro. 4 } & \multicolumn{2}{l|}{ Pro. 5 } \\
\cline { 2 - 11 } & X & Y & X & Y & X & Y & X & Y & X & Y \\
\hline Frame №1 & 73 & 101 & 355 & 148 & 424 & 242 & 173 & 360 & 669 & 382 \\
\hline Frame №2 & 64 & 117 & 357 & 141 & 430 & 242 & 180 & 369 & 663 & 381 \\
\hline Frame №3 & 81 & 112 & 369 & 140 & 432 & 242 & 170 & 372 & 682 & 383 \\
\hline Frame №4 & 73 & 108 & 354 & 156 & 437 & 241 & 172 & 367 & 665 & 383 \\
\hline
\end{tabular}

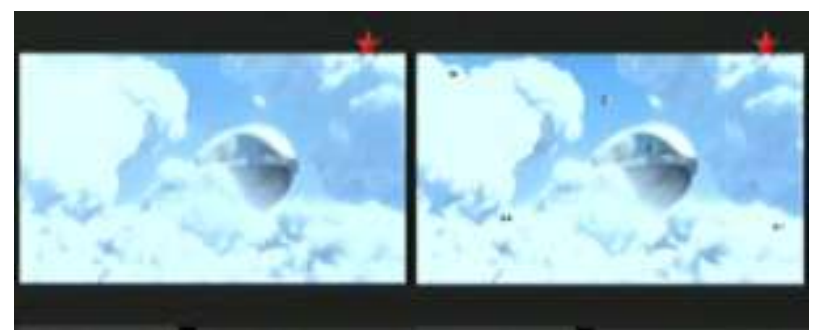

a)

b)

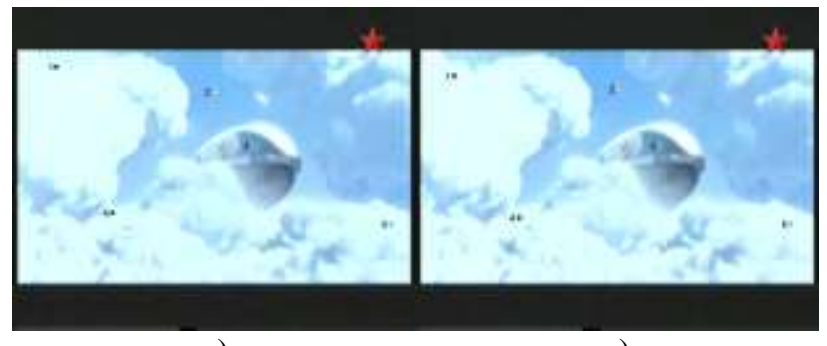

v)

g)

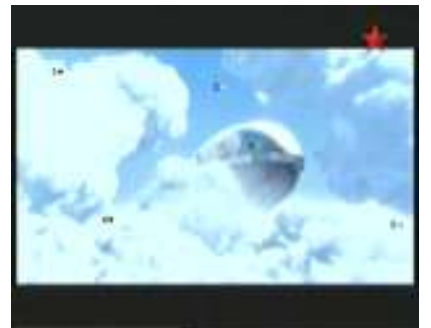

d)

Fig -1.The frames for the story "airship": the source (a), the defective block №1 (b), the defective frame №2 (v), the defective frame №3 (g), the defective frame №4 (d)

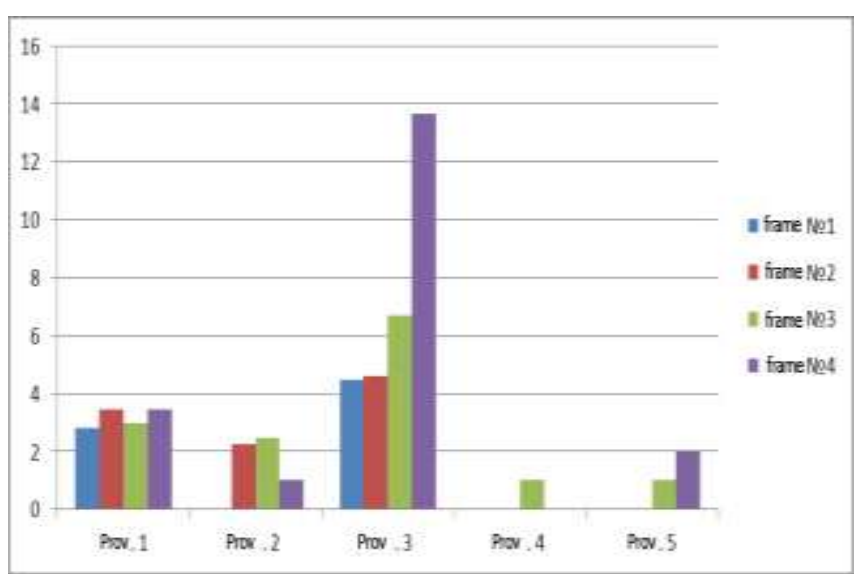

Fig -2:RMSD diagram for the "Airship" plot

Table -2: RMSD to "airship" plot

\begin{tabular}{|l|l|l|l|l|l|}
\hline & Province. 1 & Pro. 2 & Pro. 3 & Pro. 4 & Pro. 5 \\
\hline Frame №1 & 2,828427125 & 0 & 4,472135955 & 0 & 0 \\
\hline Frame №2 & 3,464101615 & 2,236067977 & 4,582575695 & 0 & 0 \\
\hline Frame №3 & 3 & 2,449489743 & 6,708203932 & 1 & 1 \\
\hline Frame №4 & 3,464101615 & 1 & 13,6381817 & 0 & 2 \\
\hline
\end{tabular}

Based on the analysis of tables, bar charts and visual evaluation obtained in the result of image processing, the conclusion can be made that defected color is recovered by $85-90 \%$, according to the structure of the video object and enclosing of its background.

\section{CONCLUSION}

In this case, if the background is more uniform, recovery will be better.

Thus, the research results showed that the use of Chebyshev polynomials of the second order allows improve effectively the visual quality of images containing disturbing objects which include different inclusion, symbols, logos and. etc. According to the experimental results obtained by specially designed software, the original image and the reconstructed image is not visually distinguishable.

Objective estimated accuracy method of restoring pixel values also indicates a low level of deviation that shows a high correcting feature of the proposed method.

\section{REFERENCES}

[1] R. Gonzalez, R. Woods, Digital Image Processing. M., Technosphere, 2005 - 1072 p

[2] Tashmanov E.B.Modernization of Processes Control Methods for Digital Image Processing //Computer Science and Information Technology Vol. 4(4), pp. $135-138$. 
[3] V.A Sadovnichy, J. Antonio, O.R Musin, O.V Seleznev, V.M Staroverov. Structural lines and critical points of the digital image // Selected topics of mathematics, mechanics and their applications. M .: Publishing House of Moscow. University Press, 1999., p 438-462.

[4] Y.I Monich, V.V Starovoytov. Quality Assessment for the analysis of digitalImages // Artificial intelligence. Minsk. - 2008. - № 4. - p.376- 386.

[5] L.S Pontryagin. Linear differential games of pursuit // Mat. Collection, 1980, T. 112, number 3, p. 307-330. 